\title{
MODELAGEM E SIMULAÇÃO DA TRANSFERÊNCIA DE MASSA AO LONGO DE MEMBRANAS CILÍNDRICAS
}

\author{
E. L. F. DE ALMEIDA ${ }^{1}$ R. L. PAGANO ${ }^{2}$ e R. R. SOUZA ${ }^{2}$ \\ ${ }^{1}$ Universidade Federal de Sergipe, Programa de Pós Graduação em Engenharia Química, \\ Laboratório de Biotecnologia Ambiental (LABAM/PEQ/UFS) \\ ${ }^{2}$ Universidade Federal de Sergipe, Departamento de Engenharia Química, Laboratório de \\ Biotecnologia Ambiental (LABAM/DEQ/UFS)
}

E-mail para contato: nosdeferreira@ yahoo.com.br

\begin{abstract}
RESUMO - Neste trabalho buscou-se entender os mecanismos de transferência de massa junto à superfície interna de membranas cilíndricas de microfiltração, onde estão associados fenômenos de difusão e convecção de uma ou mais espécies químicas presentes em uma suspensão. As espécies químicas presentes na suspensão são separadas com base nas diferenças entre os tamanhos das partículas em relação ao diâmetro médio de poros da membrana. A força motriz utilizada no processo de separação é a pressão transmembranar a que a membrana é submetida. Modelos matemáticos capazes de representar o perfil de concentração do soluto ao longo da membrana foram utilizados e resolvidos utilizando o método das linhas associado ao método das diferenças finitas com o objetivo de descrever, de uma forma geral, o comportamento de substâncias químicas, especialmente de enzimas, ao longo de membranas cilíndricas (hollow fibers ou membranas capilares ou membranas tubulares). O processo de filtração tangencial foi estudado operando em regime transiente e estacionário. Os dados obtidos com as soluções do modelo foram comparados com os dados experimentais obtidos da literatura para as enzimas $\alpha$ e $\beta$ amilase a partir do malte de milho (Zea mays) e verificou-se que o método numérico das linhas associado ao das diferenças finitas, utilizado para resolver o modelo numérico, produziu resultados compatíveis com os dados experimentais e são representativos do fenômeno estudado.
\end{abstract}

\section{INTRODUÇÃO}

Uma membrana é, basicamente, uma barreira que separa duas fases e que restringe, total ou parcialmente, o transporte de uma ou várias espécies químicas presentes nas fases. Como os processos com membranas são, em sua grande maioria, atérmicos, a força motriz que possibilita o transporte e expresso, apenas, em termos do gradiente de pressão e de concentração. O maior obstáculo inerente ao processo de separação por membranas está no declínio do fluxo de permeado ou fluxo transmembranar que ocorre devido ao acúmulo e à incrustação de partículas na superfície permeável da membrana.

A operação de instabilidade no processo de filtração tangencial, em que é aplicada uma pressão muito maior do que a pressão que pode ser absorvida pela camada de concentração de 
polarização chamada pressão crítica tem como conseqüência o bloqueio rápido dos poros e a formação de uma camada de bolo conforme trabalho de SONG (1998).

MARCOS et al. (2009) apresentaram um modelo transiente para simular numericamente o fluxo e a concentração em uma unidade de ultrafiltração. O modelo CFD desenvolvido para resolver a equação de Navier-Stokes em duas dimensões e a equação de conservação de massa para condições transientes e foi resolvido através do método dos elementos finitos.

A constrição do poro ocorre no início e corresponde à redução da estrutura interna do poro, posteriormente ocorre o bloqueio do poro, impedindo que haja mais incrustações junto à estrutura interior e finalmente a formação do bolo que reduz o fluxo em estágios mais adiantados de filtração como mostrado em DUCLOS-ORSELLO et al.(2006).

Neste estudo, buscou-se ampliar o conhecimentos com a geração de um modelo computacional que pudesse ser aplicado na simulação do processo de filtração por membranas e validado por dados experimentais.

\subsection{Objetivos}

Este trabalho centrou seu objetivo em realizar a modelagem e simulação do processo de separação de espécies químicas por membranas cilíndricas operando em regime transiente e estacionário, dando ênfase no perfil de concentração de enzimas.

\section{METODOLOGIA}

Utilizou-se um modelo matemático formado por equações de conservação de massa e conservação das espécies químicas. O modelo foi implementado em linguagem de programação FORTRAN 6.6, e resolvido utilizando o método explícito das linhas associado ao método das diferenças finitas para os regimes transiente e permanente.

Durante o regime transiente a variação da concentração ao longo de uma membrana cilíndrica pode ser descrita pela equação geral em termos de $w_{A}$ para $\rho D_{A, B}$ constante:

$$
\begin{aligned}
\rho \frac{\partial w_{A}}{\partial t}=\rho D_{A, B} & {\left[\frac{1}{r} \frac{\partial}{\partial r}\left(r \frac{\partial w_{A}}{\partial r}\right)+\frac{1}{r^{2}} \frac{\partial^{2} w_{A}}{\partial \theta^{2}}+\frac{\partial^{2} w_{A}}{\partial z^{2}}\right] } \\
& -\rho\left(v_{r} \frac{\partial w_{A}}{\partial r}+\frac{v_{\theta}}{r} \frac{\partial w_{A}}{\partial \theta}+v_{z} \frac{\partial w_{A}}{\partial z}\right)+r_{A}
\end{aligned}
$$

As considerações feitas foram de que o efeito difusivo e convectivo na direção angular $\theta$, o efeito difusivo na direção z; o efeito convectivo na direção r eram todos despreziveis; não ocorrência de reação química e, portanto, não havia geração nem consumo da espécie A.

A admensionalização das variáveis da Equação 1 resultou em:

$$
\frac{\partial \phi}{\partial \varphi}=\frac{D_{A, B} \tau}{R^{2}}\left[u \frac{\partial^{2} \phi}{\partial u^{2}}+\frac{\partial \phi}{\partial u}\right]-\frac{v_{z}}{L} \frac{\partial \phi}{\partial \epsilon}
$$

Condições de contorno:

$$
\begin{array}{ll}
u=0, & \frac{\partial \phi}{\partial u}=0 \\
u=1, & \frac{-D_{A, B}\left(\phi_{N_{r}}-\phi_{N_{r}-1}\right)}{d u}=k\left(\phi_{N_{r}}-\phi_{\infty}\right)
\end{array}
$$




$$
\begin{array}{ll}
\epsilon=0, & \phi=1 \\
\varphi=0, & \frac{\partial \phi}{\partial \varphi}=0
\end{array}
$$

Através do método das linhas associado ao método das diferenças finitas explícito, foi gerada aproximações para as derivadas da variável $\phi$ que aparece na Equação 2 nos pontos ou nós discretos $u$ e $\epsilon$. Utilizando-se apenas os valores de $\phi$ nos nós discretos $\phi_{u} \mathrm{e}$ $\phi_{\epsilon}$, substituíram-se as aproximações $\dot{\phi}_{u}, \ddot{\phi}_{u}$ e $\dot{\phi}_{\epsilon}$, na Equação 1 admensionada:

$$
\begin{gathered}
\frac{\partial \phi_{k}}{\partial \varphi}=\sigma\left[u_{i}\left(\frac{\phi_{i+1, j}-2 \phi_{i, j}+\phi_{i-1, j}}{\Delta u^{2}}\right)+\frac{\phi_{i+1, j}-\phi_{i-1, j}}{2 \Delta u}\right] \\
-\frac{v_{z}}{L}\left(\frac{\phi_{i, j+1}-\phi_{i, j-1}}{\Delta \epsilon}\right)
\end{gathered}
$$

Os índices $i=1,2,3, \ldots, \mathrm{Nr}$ e $j=1,2,3, \ldots, \mathrm{Nz}$ representam a localização dos pontos ou nós da malha de discretização em $u$ e $\epsilon$ respectivamente, o índice $k=1,2,3, \ldots$, Nt representa o espaço de tempo entre duas iterações consecutivas em $\varphi$.

Para $\mathrm{Nr}=6 \mathrm{e} \mathrm{Nz}=50$, a equação 7 foi discretizada em uma malha de 300 nós igualmente espaçados.

Durante o regime permanente, a equação de balanço de massa para o escoamento de um fluido coloidal por uma membrana cilíndrica porosa em coordenadas cilíndricas foi representada pela equação:

$$
v_{z} \frac{\partial C_{A}}{\partial z}=\frac{k T}{6 \pi \mu a_{p}}\left[\frac{1}{r} \frac{\partial}{\partial r}\left(r \frac{\partial C_{A}}{\partial r}\right)\right]
$$

A Equação (8) foi representada com derivadas em relação à $z$ considerando a variável $u$ sendo $u=r^{2}$. Considerou-se o fluxo difusivo que chega à parede interna como sendo igual ao fluxo convectivo que sai na parede externa da membrana. Para simplificar e auxiliar na comparação dos resultados, realizou-se a admensionalização das variáveis da Equação (8):

$$
\frac{\partial \phi}{\partial \epsilon}=\frac{4 D_{A, B} L}{R^{2} v_{\epsilon}}\left(u \frac{\partial^{2} \phi}{\partial u^{2}}+\frac{\partial \phi}{\partial u}\right)
$$

Condições de contorno:

$$
\begin{array}{ll}
\epsilon=0, & \phi=1 \\
u=0, & \frac{\partial \phi}{\partial u}=0 \\
u=N r, & D_{A, B} \frac{\partial \phi_{N r}}{\partial u}=k\left(\phi_{N r}-\phi_{\infty}\right)
\end{array}
$$

Foram geradas aproximações para as derivadas da variável dependente $\phi$ em nós discretos $u$; isto é, utilizando-se apenas os valores de $\phi$ nestes pontos discretos $\phi_{u}$. Substituíram-se as aproximações $\dot{\phi}_{u}$ e $\ddot{\phi}_{u}$, na Equação 9 gerando o sistema de equações diferenciais ordinárias dadas por: 


$$
\frac{\partial \phi_{j}}{\partial \epsilon}=\sigma\left(u \frac{\phi_{i+1}-2 \phi_{i}+\phi_{i-1}}{(\Delta u)^{2}}+\frac{\phi_{i+1}-\phi_{i-1}}{2 \Delta u}\right)
$$

Em que $N_{r}$, é o número de pontos ou nós de discretização na direção radial $i=1,2,3, \ldots, N_{r}$ e $N_{z}$ é o número de pontos ou nós de discretização na direção axial $j=1,2,3, \ldots, N_{z}$. Para $N_{r}=6$ e $N_{z}=50$, a região retangular correspondente a metade da seção meridiana do cilindro foi discretizada em uma malha de cinco pontos ou nós entre $r=0$ e $r=$ $R$ e 50 pontos ou nós de $z=0$ a $z=L$. Considerou-se que a concentração no meio externo à membrana é igual a zero, ou seja, $\phi_{\infty}=0$, no início e durante a filtração.

A resolução pelo método das linhas associado ao método das diferenças finitas para o regime transiente utilizando membranas cilíndrica possibilitou a comparação com dados experimentais obtidos do trabalho de SEVERO JÚNIOR (2005) realizado em um módulo de membranas cilíndricas, com o fluxo de alimentação tangencial. Foi utilizado o modelo da membrana AMICON- H1MP01-43, com área de $0,010 \mathrm{~m}^{2}$ e tamanho dos poros igual a 0,1 $\mu \mathrm{m}$ com a finalidade de recuperar e concentrar enzimas $\alpha$ e $\beta$ amilases a partir do malte de milho (Zea mays). O valor para a difusividade foi obtido do trabalho de SCHAUSBERGERA et al. (2009) para a proteína BSA. A relação de valores dos parâmetros são mostrados na Tabela 1.

Tabela 1 - Relação de parâmetros utilizados

\begin{tabular}{lccc}
\hline \multicolumn{1}{c}{ PARÂMETRO } & DESCRIÇÃO & $\begin{array}{c}\text { VALOR } \\
\text { EXPERIMENTAL }\end{array}$ & $\begin{array}{c}\text { VALOR } \\
\text { UTILIZADO NO } \\
\text { MODELO }\end{array}$ \\
\hline $\begin{array}{l}\text { Concentração na entrada } \\
\text { da membrana }\end{array}$ & $C_{0}$ & $100,63 \mathrm{~g} / \mathrm{mL}$ & $100 \mathrm{~g} / \mathrm{mL}$ \\
Raio da membrana & $R$ & $9,7.10^{-3} \mathrm{~m}$ & $9,0.10^{-3} \mathrm{~m}$ \\
Comprimento do tubo & $L$ & $10^{-1} \mathrm{~m}$ & $10^{-1} \mathrm{~m}$ \\
Difusividade & $D$ & --- & $6,7.10^{-10} \mathrm{~m}^{2} / \mathrm{s}$ \\
Velocidade axial & $v_{z}$ & $2,6.10^{-1} \mathrm{~m} / \mathrm{s}$ & $2,6.10^{-1} \mathrm{~m} / \mathrm{s}$ \\
Coeficiente de convecção & $k_{\text {ext }}$ & --- & $6.10^{-1} \mathrm{~m} / \mathrm{s}$ \\
+ resistências & & & \\
\hline
\end{tabular}

\section{RESULTADOS E DISCUSSÃO}

Os gráficos indicam como varia a concentração de soluto no interior da membrana em pontos discretos da malha. Cada ponto ou nó da malha é formado pela interseção entre uma seção transversal (radial) $u$ e uma seção longitudinal (axial) $\epsilon$.

Os valores de concentração de soluto representado por $\phi$, em cada nó da malha de discretização apresentam um perfil único para cada tempo de filtração, ou seja, diferentes perfis são verificados para diferentes tempos de filtração, representado por $\varphi$.

As partículas que se encontram distantes da parede interna ou mais próximas do centro da membrana estão menos sujeitas à camada de partículas agregadas que compõem a parede interna da membrana o que faz com que tenham maior mobilidade e assim se movimentem facilmente de um ponto a outro dentro da membrana. As partículas que se encontram próximas à superfície da parede interna da membrana estão bem próximas umas das outras 
dificultando a mobilidade, o que resulta em um perfil de concentração com menos oscilação que as partículas próximas ao centro. $\mathrm{O}$ regime transiente pode ser analisado nos gráficos exibidos na Figura 1 que mostra os perfis de concentração do soluto no interior da membrana para cada tempo de filtração.
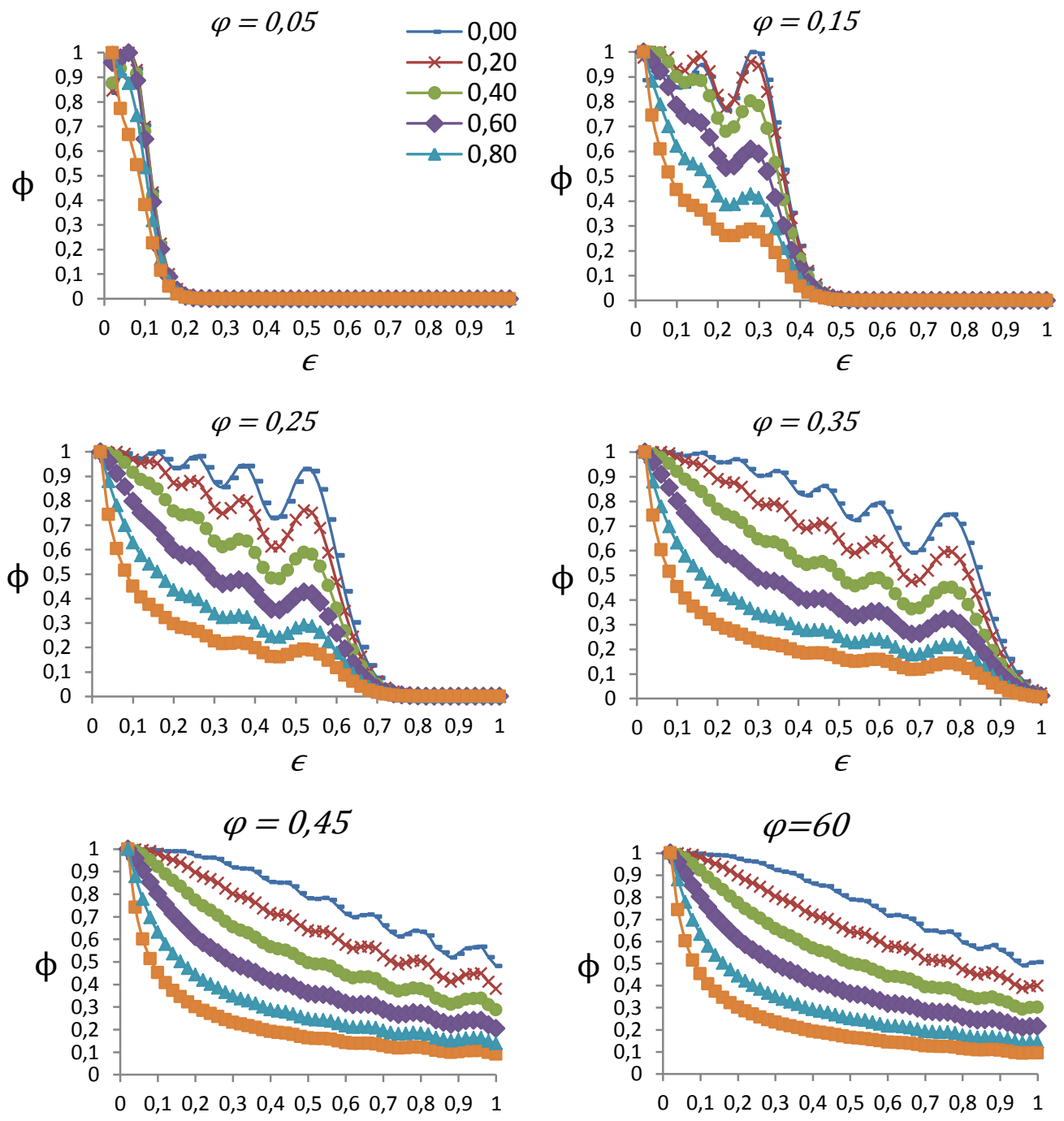

$\epsilon$

Figura 1 - Perfis de concentração do soluto $\phi$ em regime transiente em diferentes raios $\mathrm{u}=0,00$; 0,$20 ; 0,40 ; 0,60 ; 0,80$ e 1,00 versus distância axial $\epsilon$, nos tempos $\varphi=0,05 ; 0,15 ; 0,25 ; 0,35$; 0,45 e 0,60 . Todas as grandezas são adimensionais.

Durante o regime permanente produziu-se gráficos bidimensionais de concentração versus comprimento da membrana para diferentes raios variando do centro para a parede interna da membrana mostrado na Figura 2. 


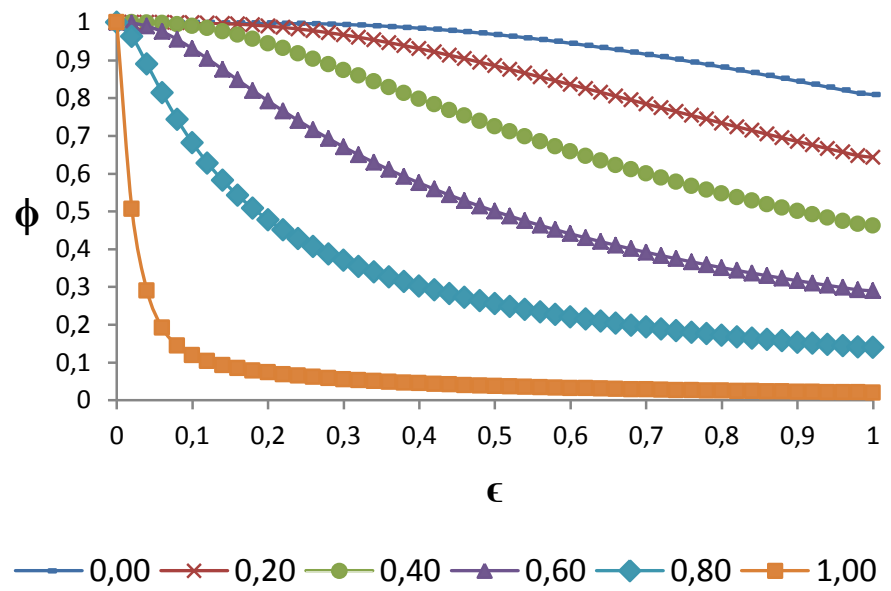

Figura 2 - Perfis de concentração de soluto em regime permanente em relação à coordenada axial $\epsilon$, para diferentes raios $\mathrm{u}=0,00 ; 0,20 ; 0,40 ; 0,60 ; 0,80$ e 1,00 .

Quanto mais próximas da parede da membrana as partículas estiverem maior a possibilidade de atravessarem os poros e serem conduzidas para o permeado.

A Figura 3 mostra um gráfico para a comparação entre dados experimentais obtidos dos trabalhos de SEVERO JÚNIOR (2005) para as enzimas $\alpha$ e $\beta$ amilases nas condições $p H$ igual a 7,00 e pressão transmembranar igual a 0,05 bar. Verificou-se que existe uma boa concordância dos dados experimentais e os dados do modelo principalmente a partir do tempo $\varphi=0,35$ em que o método é mais estável.

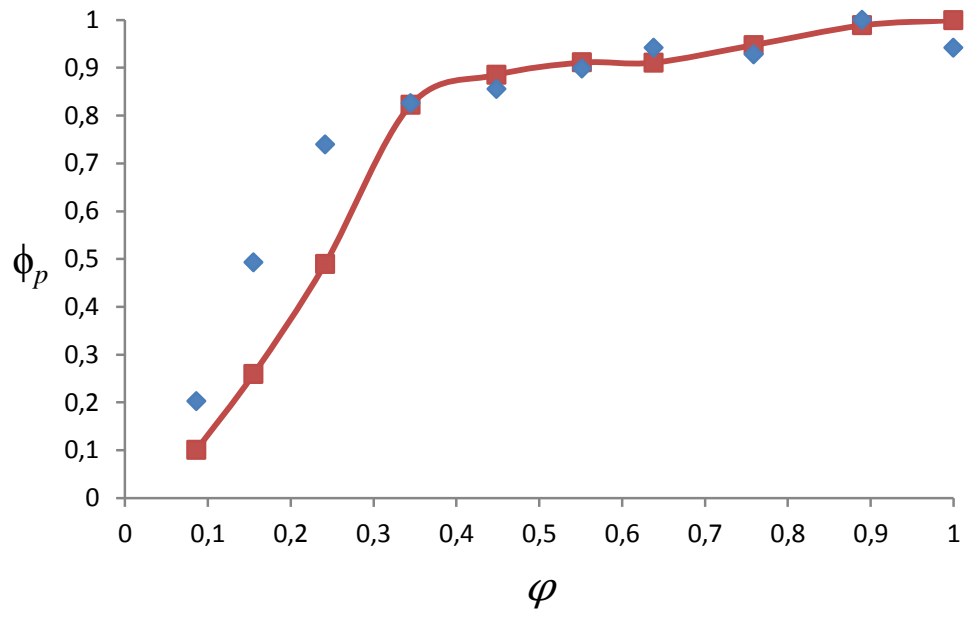

- Severo Júnior (2005) - $\rightarrow$-Solução numérica

Figura 3 - Comparação entre a solução numérica e os dados experimentais para a concentração do soluto de permeado em função do tempo das enzimas $\alpha$ e $\beta$ amilase. Todas as grandezas são adimensionais.

\section{CONCLUSÃO}

A resolução do modelo forneceu um perfil da concentração da espécie química ao longo de toda a membrana nas posições radial e axial, possibilitando identificar os fatores 
e/ou variáveis operacionais que limitam o processo de transferência do componente do seio da solução para o exterior da membrana.

Ressalta-se que o método das linhas associado ao método das diferenças finitas utilizados para resolver o sistema de equações geradas para cada ponto apresentou-se satisfatório para o caso específico para as enzimas $\alpha$ e $\beta$ amilases.

\section{NOMENCLATURA}

As dimensões são dadas em termos de massa (M), de comprimento (L), de tempo (t), de temperatura (T) e adimensionais (-)

$a_{p} \quad$ Diâmetro médio da partícula

$a \quad$ Taxa de bloqueio do poro

$C_{A} \quad$ Concentração mássica da espécie $A$

$D_{A, B} \quad$ Difusividade binária para o sistema $A-B$

$k \quad$ Constante de Boltzmann

L Comprimento total da membrana

$r$

$\sqrt{x^{2}+y^{2}}=$ Coordenada radial em coordenadas cilíndricas

$r_{A} \quad$ Taxa mássica de produção da espécie $A$ por reação química.

$R \quad$ Raio da membrana cilíndrica

$t \quad$ Tempo

T Temperatura absoluta

u $\quad(r / R)^{2}=$ Coordenada radial adimensional

$v_{z} \quad$ Componente da velocidade mássica relativa ao eixo coordenado z fixo

$w_{A} \quad$ Fração mássica da espécie $A$

z $\quad$ Coordenada axial em coordenadas cilíndricas

Letras gregas:

$\Phi$

$$
C_{A} / C_{0}=\text { Concentração mássica da espécie } A \text { adimensional }
$$


$\dot{\Phi}_{u} \quad$ Primeira derivada de $\Phi$ em relação à variável $u$

$\ddot{\Phi}_{u} \quad$ Segunda derivada de $\phi$ em relação à variável $u$

$\dot{\Phi}_{\epsilon} \quad$ Primeira derivada de $\phi$ em relação à variável $\epsilon \quad$ (-)

$\begin{array}{ll}\Phi_{N r} & C_{A} / C_{N r}=\text { Concentração mássica da espécie } A \text { adimensional na } \\ \text { parede interna do cilindro }\end{array}$

$\phi \quad$ Fração volumétrica

$\phi_{\infty} \quad$ Fração volumétrica máxima na superfície da membrana $\quad(-)$

$\theta \quad \operatorname{arctg}(y / x)=$ ângulo em coordenadas cilíndricas

$\mu \quad$ Viscosidade dinâmica $\quad$ (MLt $\left.{ }^{-1}\right)$

$\rho \quad$ Densidade do fluido $\quad\left(\mathrm{ML}^{-3}\right)$

$\tau \quad$ Tempo total de filtração $\quad$ (t)

$\varphi \quad t / \tau=$ tempo adimensional

$\epsilon \quad \begin{aligned} & z / L=\text { Concentração axial em coordenada cilíndricas } \\ & \text { adimensional }\end{aligned}$

\section{REFERÊNCIAS}

DUCLOS-ORSELLO, C.; LI, W.; HO, C.C.; A three mechanism model to describe fouling of microfiltration membranes. J. Membr. Sci., v. 280, p. 856-866, 2006.

MARCOS, B.; MORESOLI, C.; SKOREPOVA, J.; VAUGHAN, B., CFD modeling of a transient hollow fiber ultrafiltration system for protein concentration. J. Membr. Sci. v. 337, p. 136-144, 2009.

SCHAUSBERGERA, P. ; NORAZMANB, N.; LIB, H.; CHENB, V.; FRIEDLA, A.; Simulation of protein ultrafiltration using CFD: Comparison of concentration polarisation and fouling effects with filtration and protein adsorption experiments. $J$. Membr. Sci. v. 337, p. 1-8, 2009.

SEVERO JÚNIOR, J. B. Recuperação de enzimas $\alpha$ e $\beta$ amilases do malte de milho (Zea mays), utilizando processos de separação por membrana. Trabalho de Conclusão de Curso em Engenharia Química da Universidade Federal de Sergipe, São Cristóvão SE, p. 32-44, 2005.

SONG, L. Flux decline in crossflow microfiltration and ultrafiltration: mechanisms and modeling of membrane fouling. J. Membr. Sci. v. 139, p. 183-200, 1998. 